\title{
Simulation Study of the Arrhythmogenic Effects of Two Missense Mutations in Human Atrial Fibrillation
}

\author{
Rebecca Belletti ${ }^{1}$, Laura Martinez Mateu ${ }^{2}$, Lucia Romero Pèrez ${ }^{1}$, Javier Saiz ${ }^{1}$ \\ ${ }^{1}$ Centro de Investigación e Innovación en Bioingenierìa, Universitat Politècnica de València, \\ Valencia, Spain \\ 2 Departamento de Teoría de la Señal y las Comunicaciones y Sistemas Telemáticos y Computación, \\ Universidad Rey Juan Carlos, Madrid, Spain
}

\begin{abstract}
Genetic mutations affecting genes encoding for ion channel protein structures have been associated with the presence of atrial fibrillation $(A F)$ in healthy individuals. The aim of this study is to model and simulate the effects of two gain-of-function mutations found in literature, T895M and T436M, and affecting the rapid delayed rectifier potassium current. Courtemanche human atrial model has been chosen to reproduce myocytes behaviour and an optimization algorithm has been employed to fit model parameters to experimental data. Single cell and tissue patch simulations have been performed to study the effects of the two mutations in control, paroxysmal and permanent $A F$ conditions, both in right and left atrium. $O D$ simulations revealed that both mutations cause an increase in $I_{K r}$ current, leading to action potential duration shortening and flattening of restitution curves, especially in presence of the mutation T895M. Initiation of a re-entrant activity in $2 D$ simulations were possible both in case of T895M and T436M. The study reports the arrhythmogenicity of the two mutants and reveals T895M having a stronger effect with respect to T436M, in particular in control rather than in paroxysmal and permanent AF conditions. Differences in the dynamics of the two mutations highlight the importance of a patientspecific approach in planning targeted drug therapies.
\end{abstract}

\section{Introduction}

Atrial fibrillation (AF) is the most common sustained cardiac arrhythmia, characterized by chaotic and irregular activations of the upper chambers of the heart. AF nowadays affects $1.5-2 \%$ of the general population and even if it is not a life threatening disease, it can lead to a significant loss of quality of life, being so a growing issue for public healthcare system [1]. Studies revealed AF can develop in individuals who are genetically predisposed and without any other cardiovascular or metabolic disorder. This form of $\mathrm{AF}$ is known as 'Lone $\mathrm{AF}$ '.
Genetic defects in genes encoding for protein structures can thus lead to an abnormal conduction of current, influencing the delicate balance of inward and outward ions flowing through cell membranes [2]. The focus of the work is studying the effects of two gain-of-function (GOF) mutations found in literature, named T895M and $\mathrm{T} 436 \mathrm{M}$, which affect the $\mathrm{KCNH} 2$ gene, encoding for the $\alpha$-subunit of rapid delayed rectifier potassium channel, $\mathrm{Kv} 11.1$, and responsible for fibrillatory events in patients affected by them [3]. The simulation study has been performed using the Courtemanche-Ramirez-Nattel (CRN) model, then modified to introduce changes in the formulation of the $\mathrm{I}_{\mathrm{Kr}}$ current. Single cells and tissue strand simulations have been carried out to assess and characterize the potential arrhythmogenicity of the two mutations in order to give useful insights for future designing of targeted patient-specific therapies.

\section{Material and Methods}

The CRN human atrial single cell model has been chosen in this study to simulate mutant and healthy behaviour of atrial myocytes [4].

\subsection{I $\quad$ Fitting Procedure}

The $\mathrm{I}_{\mathrm{Kr}}$ formulation of the $\mathrm{CRN}$ model has been adjusted to introduce changes in the dynamics of the channel able to reproduce the mutations' behaviour. A sensitivity analysis has been therefore performed to study the effects of each parameter and to avoid a redundant parameterization. The final configuration of parameters is shown below in Equation 1 for either mutation.

A) $\mathrm{T} 895 \mathrm{M}$

$$
I_{K r}=\frac{P_{1} \cdot g_{K r} \cdot C_{m} \cdot x_{R} \cdot\left(V-E_{K}\right)}{1+e^{\frac{V+15}{22.4}}}
$$




$$
\begin{gathered}
\alpha_{\mathrm{x}(\mathrm{r})}=0.0003 \cdot \frac{(\mathrm{V}+14.1)}{1-\mathrm{e}^{\frac{\mathrm{V}+14.1}{-5 \cdot \mathrm{P}_{2}}}} \\
\beta_{\mathrm{x}(\mathrm{r})}=\mathrm{P}_{3} \cdot 7.3898 \cdot 10^{-5} \cdot \frac{(\mathrm{V}-3.3328)}{\mathrm{e}^{\frac{\mathrm{V}-3.3328}{5.1237 \cdot \mathrm{P}_{4}}}-1}
\end{gathered}
$$

B) $\mathrm{T} 436 \mathrm{M}$

$$
\begin{gathered}
I_{\mathrm{Kr}}=\frac{\mathrm{P}_{5} \cdot \mathrm{g}_{\mathrm{Kr}} \cdot \mathrm{C}_{\mathrm{m}} \cdot \mathrm{x}_{\mathrm{R}} \cdot\left(\mathrm{V}-\mathrm{E}_{\mathrm{K}}\right)}{1+\mathrm{e}^{\frac{\mathrm{V}+15}{22.4}}} \\
\alpha_{\mathrm{x}(\mathrm{r})}=0.0003 \cdot \frac{(\mathrm{V}+14.1)}{1-\mathrm{e}^{\frac{\mathrm{V}+14.1}{-5}}} \\
\beta_{\mathrm{x}(\mathrm{r})}=\mathrm{P}_{6} \cdot 7.3898 \cdot 10^{-5} \cdot \frac{\left(\mathrm{V}-3.3328+\mathrm{P}_{7}\right)}{\mathrm{e}^{\frac{\mathrm{V}-3.3328+\mathrm{P}_{7}}{5.1237 \cdot \mathrm{P}_{8}}}-1}
\end{gathered}
$$

Equation 1. $\mathrm{I}_{\mathrm{Kr}}$ formulation with parameters to adjust experimental data from mutation A) T895M and B) T436M

The CRN model differential equations were solved numerically using a variable-step solver (ode15s) and the Nelder-Mead optimization algorithm was executed in Matlab (MathWorks, Inc.) to accomplish the parameters estimation, minimizing the cost function defined as a linear combination of weighted root-mean-squared differences between target experimental data and simulated ones [5]. Parameters have been tuned accounting for relative changes within healthy conditions and experimental data affected by mutations.

\subsection{Single cell simulations}

The new formulations of the $\mathrm{I}_{\mathrm{Kr}}$ have been integrated in the CRN model along with acetylcholine current formulation $([\mathrm{ACh}]=0.005 \mu \mathrm{M})$ according to [6]. Electrical remodelling and atrial heterogeneity are taken into account by varying ionic currents to reproduce control (C), paroxysmal (pAF) and persistent atrial fibrillation (peAF) conditions, both in right (RA) and left atrium (LA) [7]. Single cell simulations were therefore performed at different basic cycle lengths (BCLs) applying a stimulus current for $2 \mathrm{~ms}$ with an amplitude of $20 \mathrm{pA} / \mathrm{pF}$. After 60 pulses of stabilization, action potentials (AP) and $\mathrm{I}_{\mathrm{Kr}}$ properties at $1 \mathrm{~Hz}$ frequency have been studied and restitution curves (APDr) have been shown to analyse the dependency to pacing frequency, in presence of genetic mutations.

\subsection{Tissue patch investigation}

Each modified version of CRN model has been applied to a 2D tissue patch of dimension $5 \times 5 \mathrm{~cm}^{2}$ with $300 \mu \mathrm{m}$ of spatial resolution. The monodomain formalism has been solved using the Elvira software [8]. Tissue patch simulations have been run applying a $2 \mathrm{~s}$ stimulus current of $100 \mathrm{pA} / \mathrm{pF}$ amplitude on a mesh with a longitudinal conductivity set at $0.0022 \mathrm{~S} / \mathrm{cm} \cdot \mathrm{pF}$ and the anisotropy ratio at 0.35 . Structural remodelling in peAF (peAF_sr) has been simulated reducing longitudinal conductivity $17 \%$ off its value. The model was considered stable after the $10^{\text {th }}$ planar pulse at BCL $1000 \mathrm{~ms}$. Vulnerable windows (VWs) and VW widths were computed for each model applying a S1-S2 cross-field protocol. Spectral and phase analysis of signals have been performed in order to compute dominant frequencies (DF) and to track rotor tips' trajectories for re-entries lasting more than $5 \mathrm{~s}$.

\section{Results}

\subsection{Fitting Procedure}

The optimization algorithm successfully achieved minimization of cost function and a good fitting of mutant experimental data (parameters values shown in Table 1).

Table 1. Parameters values for T895M and T436M mutations.

\begin{tabular}{ccccc}
\hline \hline \multicolumn{2}{c}{ T895M } & & \multicolumn{2}{c}{ T436M } \\
\hline \hline $\mathrm{P}_{1}$ & 2.055 & & $\mathrm{P}_{5}$ & 1.384 \\
$\mathrm{P}_{2}$ & 0.945 & & $\mathrm{P}_{6}$ & 0.621 \\
$\mathrm{P}_{3}$ & 0.798 & & $\mathrm{P}_{7}$ & 1.176 \\
$\mathrm{P}_{4}$ & 0.996 & & $\mathrm{P}_{8}$ & 0.995 \\
\hline \hline
\end{tabular}

Figure 1 depicts activating current, tail current and fast time constant values obtained from simulations compared to target experimental data with respect to voltages. As shown, the sets of parameters found adjust quite accurately target data.
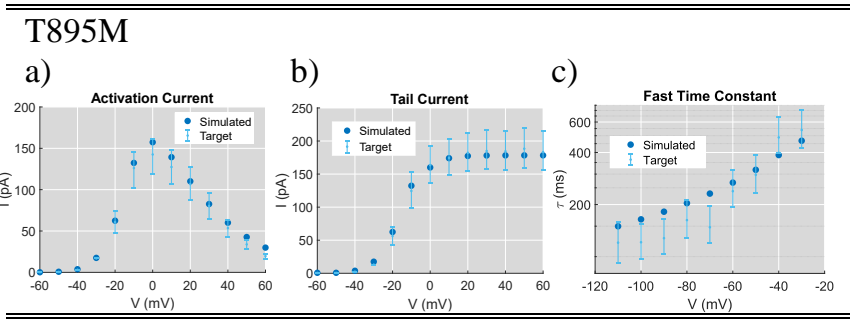

\section{$\mathrm{T} 436 \mathrm{M}$}
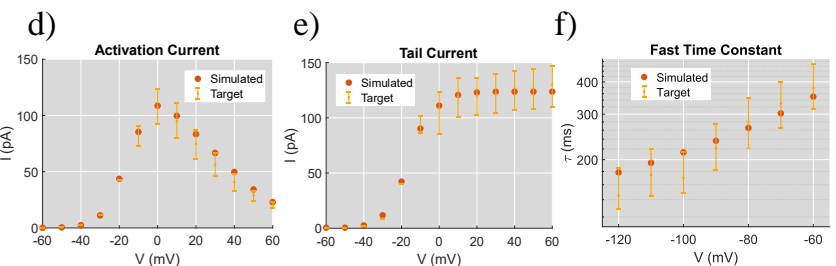

Figure 1. Comparison between target (light blue: T895M, light orange: $\mathrm{T} 436 \mathrm{M}$ ) and simulated (dark blue: $\mathrm{T} 895 \mathrm{M}$, dark orange: T436M) data.

\subsection{Single Cell Simulations}


Human atrial single cell simulations were run introducing parameters in Table 1 in the $\mathrm{I}_{\mathrm{Kr}}$ formulation of the CRN model. APs, $\mathrm{I}_{\mathrm{Kr}}$ time courses and APDr of both mutations are compared and shown with respect to their respective wild-type (WT) cases. WT is here defined as the model not affected by any of the mutations and including $\mathrm{C}, \mathrm{pAF}$ and peAF, as well as RA and LA remodelling. In every case analysed, the presence of either of the mutations caused a higher peak in the rapid potassium current; results showed T895M mutation has a higher impact in the channel dynamics, while the effect of the mutation T436M is slightly less stronger. AP duration at $90 \%$ of repolarization $\left(\mathrm{APD}_{90}\right)$ shortened in general as a consequence of higher peak current values, but its values were also affected by electrical remodelling where present. APDr showed a progressive flattening going from $\mathrm{C}$ to peAF, through pAF (Figure 2-4).

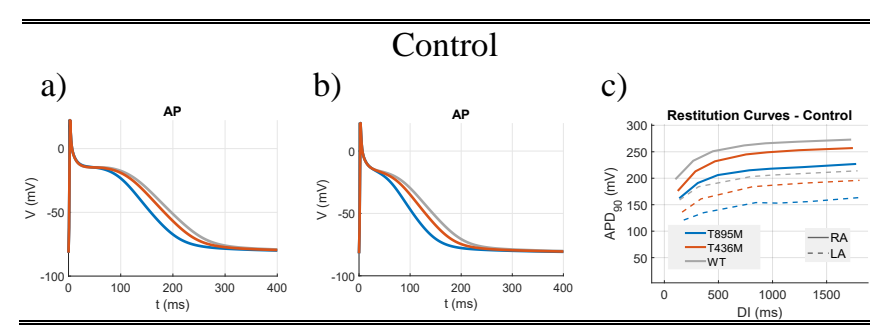

Figure 2. Action potentials in a) RA and b) LA, and c) APDr for RA (solid lines) and LA (dashed lines) for WT (grey), T895M (blue) and T436M (orange) cases in control conditions.

In RA C conditions, $\mathrm{APD}_{90}$ shortened of about $18 \%$ for $\mathrm{T} 895 \mathrm{M}$ and $7 \%$ for $\mathrm{T} 436 \mathrm{M}$, with respect to the WT value, due to an increase of the $\mathrm{I}_{\mathrm{Kr}}$ peak value $(70 \%$ and $19 \%$ for $\mathrm{T} 895 \mathrm{M}$ and $\mathrm{T} 436 \mathrm{M}$, respectively). In $\mathrm{LA}, \mathrm{I}_{\mathrm{Kr}}$ peak value increased by $66 \%$ and $\mathrm{APD}_{90}$ shortened by $24 \%$ due to the T895M mutation, with respect to WT case. T436M instead led to an $\mathrm{I}_{\mathrm{Kr}}$ peak increase of $19 \%$ and an $\mathrm{APD}_{90}$ shortening of $19 \%$ compared to the WT value. As shown in Figure 2c), APDr are shifted to lower APD 90 values for the two mutations, respect to the WT case, both in RA and LA. In addition, the maximum slope value for T895M was significantly reduced, by $20 \%$ in RA and by $43 \%$ in LA, while $\mathrm{T} 436 \mathrm{M}$ did not provoke significant changes. In RA pAF, results are the same as in RA C conditions since no changes were needed to account for electrical remodelling (Figure 3a). In LA, T895M shortened $\mathrm{APD}_{90}$ values by $18 \%$ due to an $\mathrm{I}_{\mathrm{Kr}}$ peak value $75 \%$ higher with respect to the WT case, while T436M causes an $\mathrm{I}_{\mathrm{Kr}}$ peak $22 \%$ higher and an $\mathrm{APD}_{90}$ shortening of about $7 \%$ (Figure 3b). In pAF conditions, APDr clearly shifted to lower values of $\mathrm{APD}_{90}$ in the LA. In addition, LA T895M yielded the lowest APD $_{90}$ values and the more flattened trend. In presence of T895M, the maximum slope was reduced by $22 \%$ in LA and by $20 \%$ in RA. Slope values were not affected by T436M mutation.

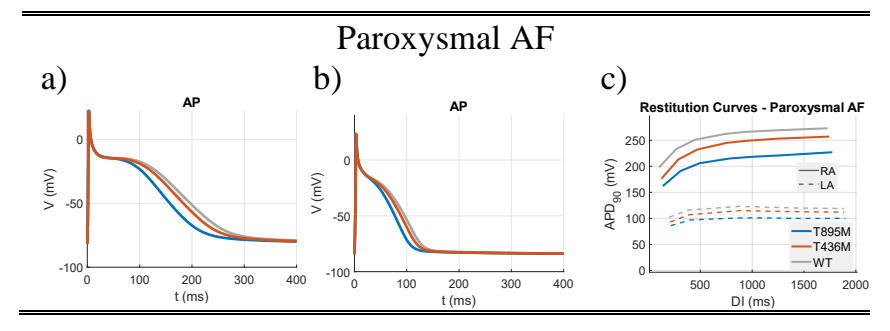

Figure 3. Action potentials in a) RA and b) LA, and c) APDr for RA (solid lines) and LA (dashed lines) for WT (grey), T895M (blue) and T436M (orange) cases in pAF conditions.

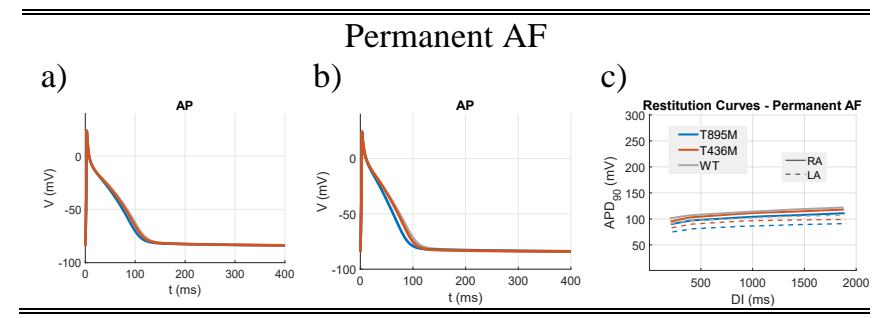

Figure 4. Action potentials in a) RA and b) LA, and c) APDr for RA (solid lines) and LA (dashed lines) for WT (grey), T895M (blue) and T436M (orange) cases in peAF conditions.

In RA peAF conditions, T895M mutation generated an $\mathrm{APD}_{90}$ shortening of $9 \%$ respect to the WT value, while T436M instead shortened $\mathrm{APD}_{90}$ almost by $3 \%$. $\mathrm{I}_{\mathrm{Kr}}$ peak increased of about $90 \%$ for $\mathrm{T} 895 \mathrm{M}$ and $28 \%$ for T436M. In LA, T895M provoked an $\mathrm{APD}_{90}$ shortening of $15 \%$ and $\mathrm{T} 436 \mathrm{M}$ of about $5 \%$ the $\mathrm{WT}$ value. $\mathrm{I}_{\mathrm{Kr}}$ peak values were $37 \%$ and $35 \%$ higher than WT case, for T895M and T436M, respectively. APDr in peAF were similar both in terms of $\mathrm{APD}_{90}$ range of values and maximum slope.

\subsection{Tissue Strand Simulations}

The study of the temporal vulnerability of the tissue revealed that both mutations are able to initiate a reentrant activity; however, no significant differences in temporal vulnerability width were found. Dominant frequency (DF) values lie in the range [9.5, 14.3] as reported in literature for subjects affected by AF; moreover, spatial distribution of DF shows that high DF areas coincides with the central areas of rotors' meandering in most of the cases [9]. In Figure 5, rotors' trajectories are shown during the $5 \mathrm{~s}-$ simulation. In $\mathrm{C}$, mutation T895M in LA favoured re-entries, maintained up to 5 seconds. The meandering area appears large, but the pattern seems quite regular. In pAF simulations, spiral waves were more stable, occupying a less extended area respect to C. In particular, rotor in T895M appeared much more regular in the shape and with a slightly smaller area respect to T436M and WT, even though in pAF T895M had less impact than in C. Finally, in peAF and peAF_sr, 
both meandering centre areas and pattern of the rotors show no differences, when comparing the two mutations to WT.

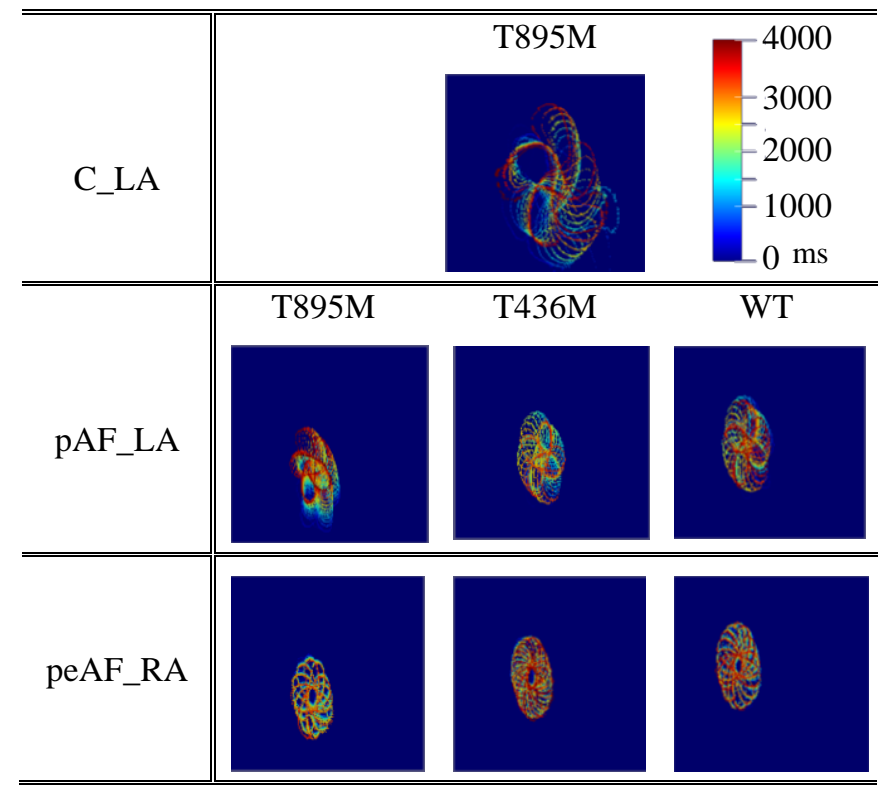

Figure 5. Rotors' tip trajectories color-coded on $\mathrm{ms}$ of simulation

\section{Discussion and Conclusions}

In this study, two genetic defects related to AF and affecting the $\mathrm{KCNH} 2$ gene encoding for $\mathrm{Kv} 11.1$ protein have been characterized and their effects have been studied in single cell and tissue patch simulations. Parameter estimation through optimization allowed a good fitting of experimental data, reproducing pathological channel dynamics. $\mathrm{C}, \mathrm{pAF}$, peAF and peAF_sr cases have been analysed. Overall, T895M mutation produces a higher peak in $\mathrm{I}_{\mathrm{Kr}}$ current and a more pronounced $\mathrm{APD}_{90}$ shortening respect to $\mathrm{T} 436 \mathrm{M}$; the effect is stronger in LA rather than in RA. Moreover, only in presence of the former mutation a reduction of the APDr maximum slope values is observed. This result is evident in $\mathrm{C}$ and $\mathrm{pAF}$ conditions, but not in peAF and peAF_sr. This may be due to the electrical remodelling counterbalancing the effect of the mutation on the system. $\mathrm{APD}_{90}$ and ERP shortening, as well as reduction in maximum slope values in APD90r, have been demonstrated to be able to generate a substrate for initiation and maintenance of spiral waves, as confirmed by 2D simulations in tissue patch reported in this study. Rotors can be initiated in every condition except for C WT in RA. As shown also in single cells, 2D simulations confirmed that T895M, in LA, has the strongest effect on the system causing initiation and progression of re-entrant activities, where difficult in WT (C), and a more stable and regular re-entry, where already possible in WT (pAF). Whole-atria and torso simulations will be necessary to further characterize the impact of the T895M and T436M mutation at organ level, helping prevent fibrillatory events through patient-specific targeted drug treatments.

\section{Acknowledgments}

I acknowledge this work to the European Union's Horizon 2020 research and innovation programme under the Marie Skłodowska-Curie gran agreement No.766082 and to Generalitat Valenciana, Prometeo programme 2020/043.

\section{References}

[1] E. Society et al., "Guidelines for the management of atrial fibrillation The Task Force for the Management of Atrial Fibrillation of the Developed with the special contribution of the European Heart Rhythm Association," 2010, doi: 10.1093/eurheartj/ehq278.

[2] J. Feghaly, P. Zakka, B. London, C. A. Macrae, and M. M. Refaat, "Genetics of Atrial Fibrillation," J. Am. Heart Assoc., pp. 1-15, 2018, doi: 10.1161/JAHA.118.009884.

[3] K. Hayashi et al., "Functional Characterization of Rare Variants Implicated in Susceptibility to Lone Atrial Fibrillation," Circ. Arrhythmia Electrophysiol., vol. 8, no. 5, pp. 1095-1104, 2015, doi: 10.1161/CIRCEP.114.002519.

[4] M. Courtemanche, R. J. Ramirez, and S. Nattel, "Ionic mechanisms underlying human atrial action potential properties: insights from a mathematical model," Am. J. Physiol. -Heart Circ. Physiol., vol. 275, pp. 301321, 1998, doi: 10.1152/ajpheart.1998.275.1.H301.

[5] B. J. A. Nelder and R. Mead, "A simplex method for function minimization," 1964.

[6] E. Grandi et al., " Human Atrial Action Potential and Ca 2+ Model ," Circ. Res., vol. 109, no. 9, pp. 10551066, 2011, doi: 10.1161/circresaha.111.253955.

[7] L. Martinez Mateu, "Mapping of the Electrical Activity of Human Atria Multiscale Modelling and Simulations" 2018, https://doi.org/10.4995/Thesis/10251/104604.

[8] E. A. Heidenreich, J. M. Ferrero, M. Doblaré, and J. F. Rodríguez,"Adaptive macro finite elements for the numerical solution of monodomain equations in cardiac electrophysiology,"Ann. Biomed. Eng., vol. 38, no. 7, pp. 2331-2345, 2010, doi:10.1007/s10439-010-9997-2.

[9] P. Sanders et al., "Spectral analysis identifies sites of high-frequency activity maintaining atrial fibrillation in humans," Circulation, vol. 112, no. 6, pp. 789-797, 2005, doi: 10.1161/CIRCULATIONAHA.104.517011.

Address for correspondence:

Rebecca Belletti

Universitat Politecnica de Valencia, Cami de Vera s/n, 46022, Valencia, Valencia, España

rbelletti@ci2b.upv.es 